\title{
On the Use of Connected Moments Expansion with Coupled Cluster Reference
}

\author{
Jozef Noga ${ }^{1}$, Ágnes Szabados ${ }^{2}$ and Péter R. Surján ${ }^{3}$ \\ ${ }^{1}$ Institute of Inorganic Chemistry, Slovak Academy of Sciences, SK-84236 Bratislava, Slovakia, \\ E-mail: noga@savba.sk \\ ${ }^{2}$ Structural Chemistry Research Group, Hungarian Academy of Sciences, Eötvös University, \\ POB 32, H-1518 Budapest, Hungary, E-mail: szabados@chem.elte.hu \\ ${ }^{3}$ Department of Theoretical Chemistry, Eötvös University, POB 32, H-1518 Budapest 112, \\ Hungary, E-mail: surjan@chem.elte.hu
}

Received: 6 December 2001 / Accepted: 18 January 2002 / Published: 31 May 2002

\begin{abstract}
We examine the possibility of introducing a new class of so called noniterative corrections to coupled cluster energies, based on the connected moments expansion (CMX). Approximate Coupled Cluster (CC) wave functions are used as reference states and then the "improved energies" can be formally obtained either by CMX in terms of the moments of CC similarity transformed Hamiltonian, or in CMX of the usual Hamiltonian. Numerical results are given for some model systems that show the superiority of the latter approach.
\end{abstract}

Keywords: Electron correlation; Coupled cluster theory; Non-iterative corrections; Connected moments expansion.

(C)2002 by MDPI, Basel, Switzerland. Reproduction for noncommercial purposes permitted. 


\section{Introduction}

Coupled cluster (CC) theory [1-3] continues to be one of the most important theoretical tools in the many-body problem, providing relatively accurate, size extensive wave functions and energies even at the level of singles (S) and doubles (D) (CCSD) [4], using a conceptually simple single reference approach (SR-CC). In spite of the fact that in the latter model a substantial part of the electron correlation effects of triply and quadruply excited determinants is included via disconnected clusters, it is well documented by now that inclusion of higher connected excitation clusters is necessary to obtain highly accurate and reliable data. This holds especially if one aims to study reactive potential energy surfaces. Though full inclusion of triples (T) via CCSDT $[5,6]$ or quadruples (Q) via CCSDTQ $[7,8]$ is possible, they are very expensive to compute. Even more expensive are the configuration interaction (CI) based CC algorithms that can treat basically any excitations up to full CI [9-12]. Approximate inclusion of triples [13-15] or quadruples [16] into the CC equations allows more favorable algorithms. These are, however, still too demanding for an iterative procedure to be applied when solving the CC equations. Experience shows that these approximate iterative solutions are not clearly superior to computationally much more effective, so called non-iterative corrections, which can be applied after some given CC equations were solved. Adjective "non-iterative" reflects the fact that approximate amplitudes of higher excitations are determined in a single step, without solving the nonlinear CC equations. Those amplitudes are used subsequently to correct the energy and/or properties.

The most popular corrections due to triples are certainly those based on CCSD, namely $\operatorname{CCSD}[\mathrm{T}]^{1}[14]$ or the slightly different $\operatorname{CCSD}(\mathrm{T})$ [17]. Those two approximations are formally correct up to the fourth order of Many Body Perturbation Theory (MBPT) for closed shell Hartree-Fock (HF), or an unrestricted HF (UHF) reference. The latter criterion was also fulfilled in a similar treatment suggested independently in order to correct the CCD energies for contributions from singles and triples, i. e. CCD+ST(CCD) [19]. However, because of the superiority of CCSD basically for the same price, this $\mathrm{CCD}+\mathrm{ST}(\mathrm{CCD})$ approximation has not found a wide use.

During the last 15 years several non-iterative corrections have been suggested and implemented, including those used for open shell systems or excited states [12,21-35]. Initial argumentation for these corrections was based on the analysis of the energy in terms of perturbation theory. The CC energy has been corrected either to include all contributions up to a certain order, or to include at least those terms of MBPT up to a certain order, that were present in the higher variant of the coupled cluster approach. For non-degenerate cases, approaches correct to 4th order work well, while to include non-dynamical correlation one often needs fifth or higher order corrections.

\footnotetext{
${ }^{1}$ Originally denoted as CCSD+T(CCSD). Abbreviation CCSD $[\mathrm{T}]$ was introduced for simplicity in [18].
} 
Unfortunately, there is no unique prescription which fifth or higher order corrections need to be included. Though, one naturally expects that contributions due to connected quadruples (which are missing in MBPT(4)) would be of great importance, there are several other contributions that are of similar magnitude [36], and moreover, there is usually a substantial cancellation between the fifth-order corrections [21, 28, 36, 37]. This, with the fact that MBPT with Møller-Plesset splitting of the Hamiltonian can (surprisingly) diverge even for non-degenerate cases [38], is certainly a drawback for high precision predictions, and at the same time a challenge for alternative treatments. Indeed, such alternative approaches have appeared very recently.

One class is represented by "renormalized" or "completely renormalized" non-iterative corrections suggested by Kowalski and Piecuch [31] which are based on the method of moments of CC equations (MMCC). Following the formalism of $\beta$ nested equations ( $\beta$-NE) [39], Kowalski and Piecuch formulated and derived the so called Fundamental Theorem of the Formalism of $\beta$ Nested Equations [30], which has been an inspiration for MMCC. The principal idea was to provide an explicit formula for a non-iterative correction to the energy obtained by an approximate SR-CC method to recover the FCI result. The authors have also shown that the general formula for the renormalized CC corrections can be as well derived by using "standard" argumentation of the CC theory, hence avoiding the formalism of $\beta$-NE. Recently, MMCC approach has been extended to excited states [32], as well as to multireference wave operator formalism [33].

Analogous type of corrections can be derived by explicitly considering the coupling between the energy and CC amplitudes in all "a posteriori" approaches [35]. At the level of renormalized $\mathrm{CCSD}[\mathrm{T}]$ or $\operatorname{CCSD}(\mathrm{T})$ the corrections are identical with those using MMCC argumentation.

Schematically, the renormalized corrections can be expressed as:

$$
\Delta E_{R}=\frac{\Delta E}{1+S}
$$

where $\Delta E_{R}$ is the renormalized energy correction, $\Delta E$ stands for the standard non-iterative correction [such as $\operatorname{CCSD}[\mathrm{T}], \operatorname{CCSD}(\mathrm{T}), \operatorname{CCSD}(\mathrm{TQ}) \ldots$... while the "renormalization term" $S$ depends on the chosen variant. From the point of view of single-reference perturbation expansion, $S$ contains at least second-order contributions. Hence, despite the corrections in various approximate variants may not be size extensive, the inextensivity error can be considered small for singlereference situations. Moreover, if quasi-degeneracy increases, "overshooting" of $\Delta E$ is reduced by the increasing denominator. As a result renormalized non-iterative corrections offer a promising way of extending the applicability of the standard corrections to a larger range of molecular geometries, as documented by recent works [40].

A different class of corrections to $\mathrm{CC}$ energies is offered by a hierarchy of corrections based on the perturbative expansion of the similarity-transformed Hamiltonian $[12,34]$ which directly follows from ideas addressed earlier [25, 41]. 
In this paper we sketch yet another possibility for non-iterative corrections that are based on the so called connected moments expansion (CMX) of the energy. For the use in molecular ab initio calculations, CMX was suggested in the late eighties by Cioslowski [42], and initially used within the framework of a single reference theory $[42,43]$ with medium success. The way of constructing CMX expansions is, however, not unique [44-46]. As demonstrated on a few examples, the expansion termed as CMX-LT [44, 45], could be superior in molecular calculations. For simple molecular systems, the idea of connected moments expansion has been also applied in variational Monte Carlo method [47].

The CMX approach was criticized by Wolinski and Pulay [48], claiming that such an approach is inappropriate for computing potential energy surfaces. According to our numerical experience with CMX, the above criticism is only relevant if one starts the CMX expansion with a single reference configuration. As soon as one has a multiconfigurational framework the problem disappears [49].

In the next section we shall briefly recapitulate the CMX theory in general and subsequently we discuss the theory within the CC framework. Finally, in Sec. 4 we present some numerical examination of the possible CMX-CC models.

\section{The Connected Moments Expansion}

The CMX expansion is derived from the Horn-Weinstein function [50]

$$
f(\eta)=\frac{\left\langle\Phi\left|\hat{H} e^{-\eta \hat{H}}\right| \Phi\right\rangle}{\left\langle\Phi\left|e^{-\eta \hat{H}}\right| \Phi\right\rangle}
$$

where $H$ is the Hamiltonian, while $\Phi$ is an arbitrary reference function having a nonzero overlap with the true ground state of $H$. This function possesses the following properties:

i) $f(0)$ is the expectation value of $\hat{H}$ with the reference function

ii) it is monotonically decreasing

iii) its Taylor expansion coefficients are the connected moments of $\hat{H}$

iv) $f(\infty)$ is the exact ground state energy.

We note that if one used the full Hamiltonian in the Hilbert space, than the exact solution of the Schrödinger equation would be recovered at $f(\infty)$, while if dealing with the second quantized Hamiltonian that corresponds to a finite basis set, $f(\infty)$ gives the full CI solution.

Based on the above properties of the Horn-Weinstein function, some time ago Cioslowski proposed a successive approximation to the ground state energy [42] termed the connected moments expansion:

$$
E_{C M X-H W}=I_{1}-\frac{I_{2}^{2}}{I_{3}}-\frac{A_{6,1}^{2}}{I_{3} A_{8,1}}-\frac{\left(A_{10,1} A_{6,1}-A_{8,1}^{2}\right)^{2}}{I_{3} A_{8,1}\left(A_{12,1} A_{8,1}-A_{10,1}^{2}\right)}-\ldots
$$




$$
\begin{aligned}
& A_{2 m, i}=I_{m+i} I_{m-i}-I_{m+i-1} I_{m-i+1} \\
& A_{2 m+1, i}=I_{m+i+1} I_{m-i}-I_{m+i} I_{m-i+1}
\end{aligned}
$$

where $I_{k}$ are the connected moments of the Hamiltonian given as

$$
\begin{aligned}
f(0)=I_{1} & =\langle\hat{H}\rangle \\
-\left(\frac{d f}{d \eta}\right)_{\eta=0}=I_{2} & =\left\langle\hat{H}^{2}\right\rangle-\langle\hat{H}\rangle^{2} \\
-\left(\frac{d^{2} f}{d \eta^{2}}\right)_{\eta=0}=I_{3} & =\left\langle\hat{H}^{3}\right\rangle-3\left\langle\hat{H}^{2}\right\rangle\langle\hat{H}\rangle+2\langle\hat{H}\rangle^{3} \\
-\left(\frac{d^{3} f}{d \eta^{3}}\right)_{\eta=0}=I_{4} & =\left\langle\hat{H}^{4}\right\rangle-4\left\langle\hat{H}^{3}\right\rangle\langle\hat{H}\rangle-3\left\langle\hat{H}^{2}\right\rangle^{2}+12\left\langle\hat{H}^{2}\right\rangle\langle\hat{H}\rangle^{2}-6\langle\hat{H}\rangle^{4} \\
-\left(\frac{d^{4} f}{d \eta^{4}}\right)_{\eta=0}=I_{5} & =\left\langle\hat{H}^{5}\right\rangle-5\left\langle\hat{H}^{4}\right\rangle\langle\hat{H}\rangle-10\left\langle\hat{H}^{3}\right\rangle\left\langle\hat{H}^{2}\right\rangle+20\left\langle\hat{H}^{3}\right\rangle\langle\hat{H}\rangle^{2} \\
& +30\left\langle\hat{H}^{2}\right\rangle^{2}\langle\hat{H}\rangle-60\left\langle\hat{H}^{2}\right\rangle\langle\hat{H}\rangle^{3}+24\langle\hat{H}\rangle^{5} .
\end{aligned}
$$

We use $\left\langle\hat{H}^{k}\right\rangle=\left\langle\Phi\left|\hat{H}^{k}\right| \Phi\right\rangle$. One easily recognizes that

$$
I_{k}=\left\langle\hat{H}^{k}\right\rangle-\sum_{i=0}^{k-2}\left(\begin{array}{c}
k-1 \\
i
\end{array}\right) I_{i+1}\left\langle\hat{H}^{k-i-1}\right\rangle=\left\langle\hat{H}^{k}\right\rangle_{C}
$$

where subscript "C" stands for connected. According to [44], we shall denote the CMX variant given by Eq. (3) as "HW". Truncation after the first, second, etc. terms defines CMX-HW(1), CMX-HW(2), etc. approximations.

The expansion Eq. (3) can be also derived using the theory of standard Padé approximants, while the above mentioned CMX-LT can be obtained using the theory of generalized Padé approximants. The latter procedure is equivalent to a derivation based on considering the Lanczos basis [51] for the Hamiltonian matrix [45]. Elegant recursive formulas are given in the latter paper for both CMX-HW and CMX-LT series. Explicitly, the first four terms in the CMX-LT expansion read:

$$
\begin{aligned}
& E_{C M X-L T}=I_{1}-\frac{I_{2}^{2}}{I_{3}}-\frac{A_{6,1}^{2}}{I_{3} A_{8,1}} \\
& -\frac{A_{6,1}\left(A_{9,1} A_{6,1}-A_{8,1} A_{7,1}\right)^{2}}{I_{3} A_{8,1}\left[A_{8,1}\left(A_{10,2} A_{6,1}-A_{8,1} A_{8,2}\right)-A_{9,1}\left(A_{9,1} A_{6,1}-A_{8,1} A_{7,1}\right)\right]}-\ldots
\end{aligned}
$$

Clearly, CMX-HW(n) and CMX-LT(n) are identical up to $\mathrm{n}=3$. 
We intend to investigate

i) whether the above expansion of the true energy is feasible if $\Phi$ is an approximate CC wave function, say CCSD; and

ii) whether a modification of the original Horn-Weinstein function, replacing the expectation values by non-symmetric matrix elements in the spirit of $\mathrm{CC}$ theory, provides meaningful results.

\section{CMX Using a Coupled Cluster Reference State}

The reference function $\Phi$ in Eq. (2) and (3) is arbitrary, hence it can also be taken as an approximate CC wave function:

$$
|\Phi\rangle=e^{T}|H F\rangle
$$

where $|H F\rangle$ is the single determinantal reference state (Fermi vacuum), typically chosen as the leading configuration in $|\Phi\rangle$. Intermediate normalization is assumed, i. e. $\langle H F \mid \Phi\rangle=1$. The global excitation cluster operator $T$ is restricted only to certain excitations with respect to $|H F\rangle$, most frequently $T=T_{1}+T_{2}$. Let us recall that in traditional $\mathrm{CC}$ approach, energy and the amplitudes of $T$ are obtained from:

$$
\begin{aligned}
E_{C C} & =\left\langle H F\left|\hat{H} e^{T}\right| H F\right\rangle=\left\langle H F\left|\hat{H} e^{T}\right| H F\right\rangle_{C}, \\
0 & =\left\langle H F_{n}\left|(\hat{H}-E) e^{T}\right| H F\right\rangle=\left\langle H F_{n}\left|\hat{H} e^{T}\right| H F\right\rangle_{C},
\end{aligned}
$$

$\left|H F_{n}\right\rangle$ denoting determinants created by $T$.

Due to the aforementioned definition of the connected moments one arrives at:

$$
\left\langle\hat{H}^{k}\right\rangle_{C}=\left\langle H F\left|e^{T^{\dagger}} \hat{H}^{k} e^{T}\right| H F\right\rangle_{C} .
$$

Clearly, $\langle\hat{H}\rangle$ is not a coupled cluster energy calculated in a traditional way. Though already in the original coupled cluster papers it is proven that an expectation value of the Hamiltonian (or any nelectron operator) in terms of the CC wave function is a connected expansion [3], such an approach is not widely used. The reason is simply that evaluation of such matrix elements is substantially complicated due to the occurrence of the (deexcitation) operator $e^{T^{\dagger}}$ in the bra functions. This results in a non-terminating, though factorizable, expansion of the expectation value. Thinking in this way, one meets the problem of determining where to terminate this expansion, since unfortunately, it consists of many mutually (almost) canceling terms [52]. An alternative way is to compute these matrix elements by determining the $\mathrm{CC}$ wave function, converting the $\mathrm{CC}$ amplitudes to CI-type coefficients. In principle the procedure involves letting the Hamiltonian act on the $e^{T}|H F\rangle$, and taking the scalar product of the two vectors. This is certainly very demanding computationally, but easy to apply as soon as a general CI program is available. We used the latter approach in obtaining the testing numerical examples reported below. 


\subsection{Modified CC-CMX formulas}

In $\mathrm{CC}$ theory it appears to be challenging to investigate a possibility of using a non-symmetric modified form of the Horn-Weinstein function, in analogy with the non-symmetric energy formula Eq. (14). Function

$$
\tilde{f}(\eta)=\frac{\left\langle H F\left|\hat{H} e^{-\eta \hat{H}} e^{T}\right| H F\right\rangle}{\left\langle H F\left|e^{-\eta \hat{H}} e^{T}\right| H F\right\rangle}
$$

shares several properties of the original Horn-Weinstein function of Eq. (2). In particular, $\tilde{f}(0)=$ $\left\langle H F\left|H e^{T}\right| H F\right\rangle=E_{C C}$ is the coupled cluster energy, and $\tilde{f}(\infty)=E_{0}$ is the exact (full CI) eigenvalue. To prove the latter statement, consider the spectral resolution of $\hat{H}$ and insert it into (17):

$$
\tilde{f}(\eta)=\frac{\sum_{i} E_{i} e^{-\eta E_{i}}\left\langle H F \mid \Psi_{i}\right\rangle\left\langle\Psi_{i}\left|e^{T}\right| H F\right\rangle}{\sum_{i} e^{-\eta E_{i}}\left\langle H F \mid \Psi_{i}\right\rangle\left\langle\Psi_{i}\left|e^{T}\right| H F\right\rangle}
$$

If both the numerator and denominator are multiplied by $e^{\eta E_{0}}$ ( $E_{0}$ the ground state), all exponents $-\eta\left(E_{i}-E_{0}\right)$, but $-\eta\left(E_{0}-E_{0}\right)$, become negative. Hence, for $\eta \rightarrow \infty$ limit, only the term with the lowest exponent $E_{0}$ survives, and we are left with

$$
\lim _{\eta \rightarrow \infty} \tilde{f}(\eta)=\frac{E_{0} e^{-\eta E_{0}}\left\langle H F \mid \Psi_{0}\right\rangle\left\langle\Psi_{0}\left|e^{T}\right| H F\right\rangle}{e^{-\eta E_{0}}\left\langle H F \mid \Psi_{0}\right\rangle\left\langle\Psi_{0}\left|e^{T}\right| H F\right\rangle}=E_{0} .
$$

Moreover, differentiating $\tilde{f}(\eta)$ with respect to $\eta$ and taking the derivatives at $\eta=0$ we obtain

$$
\begin{aligned}
-\tilde{f}^{\prime}(0)=I_{2}^{C C} & =\left\langle H F\left|\hat{H} \hat{H} e^{T}\right| H F\right\rangle-\left\langle H F\left|\hat{H} e^{T}\right| H F\right\rangle\left\langle H F\left|\hat{H} e^{T}\right| H F\right\rangle \\
& =\left\langle H F\left|e^{T} e^{-T} \hat{H} e^{T} e^{-T} \hat{H} e^{T}\right| H F\right\rangle-\left\langle H F\left|e^{T} e^{-T} \hat{H} e^{T}\right| H F\right\rangle\left\langle H F\left|e^{T} e^{-T} \hat{H} e^{T}\right| H F\right\rangle \\
& =\left\langle H F\left|\bar{H}^{2}\right| H F\right\rangle-\langle H F|\bar{H}| H F\rangle^{2} \\
& =\left\langle H F\left|\bar{H}^{2}\right| H F\right\rangle_{C} .
\end{aligned}
$$

In the final steps of Eq. (20) we have used $\bar{H}$ for the similarity transformed Hamiltonian:

$$
\bar{H}=e^{-T} \hat{H} e^{T}
$$

and, we used the fact that

$$
\langle H F| e^{T} e^{-T}=\langle H F| e^{-T} .
$$

Analogously one can show that, denoting the derivatives of $\tilde{f}(\eta)$ by parenthesized superscripts,

$$
-\tilde{f}^{(k-1)}(0)=I_{k}^{C C}=\left\langle H F\left|\bar{H}^{k}\right| H F\right\rangle_{C} .
$$

In fact, one can now easily see that the function of Eq. (17) is actually equivalent to:

$$
\tilde{f}(\eta)=\frac{\langle H F|\bar{H} \exp (-\eta \bar{H})| H F\rangle}{\langle H F|\exp (-\eta \bar{H})| H F\rangle}
$$


Table 1: Deviations of the CMX energies (in $\mathrm{m} E_{\mathrm{h}}$ ) from the full CI energies (in parenthesis) for beryllium atom with different basis sets.

\begin{tabular}{|c|c|c|c|c|c|c|}
\hline & \multicolumn{6}{|c|}{ Reference function } \\
\hline & \multicolumn{2}{|c|}{ CCSD } & \multirow[t]{2}{*}{$\mathrm{CISD}^{\mathrm{c}}$} & \multicolumn{2}{|c|}{ CCSDT } & \multirow[t]{2}{*}{ CISDT $^{\mathrm{C}}$} \\
\hline & $\mathrm{CC}^{\mathrm{a}}$ & $\mathrm{XCC}^{\mathrm{b}}$ & & $\mathrm{CC}^{\mathrm{a}}$ & $\mathrm{XCC}$ & \\
\hline \multicolumn{7}{|c|}{$3-21 \mathrm{G} \quad\left(-14.531444 E_{\mathrm{h}}\right)$} \\
\hline CMX-HW(1) & 0.028 & 0.028 & 0.048 & 0.002 & 0.000 & 0.023 \\
\hline CMX-HW(2) & 0.028 & 0.003 & 0.005 & 0.002 & 0.000 & 0.000 \\
\hline CMX-HW(3) & 0.032 & 0.000 & 0.000 & 0.001 & 0.000 & 0.000 \\
\hline CMX-HW(4) & 0.024 & 0.000 & 0.000 & -0.001 & 0.000 & 0.000 \\
\hline CMX-LT(4) & 0.018 & 0.000 & 0.000 & 0.000 & 0.000 & 0.000 \\
\hline \multicolumn{7}{|c|}{$6-311 \mathrm{G} \quad\left(-14.632864 E_{\mathrm{h}}\right)$} \\
\hline CMX-HW(1) & 0.189 & 0.190 & 1.492 & 0.004 & 0.001 & 1.312 \\
\hline CMX-HW(2) & 0.189 & 0.023 & 0.129 & 0.004 & 0.000 & 0.083 \\
\hline CMX-HW(3) & 0.150 & 0.003 & 0.054 & 0.003 & 0.000 & 0.036 \\
\hline CMX-HW(4) & -0.234 & 0.001 & -0.010 & 0.002 & 0.000 & 0.038 \\
\hline CMX-LT(4) & -0.197 & 0.001 & -0.012 & 0.003 & 0.000 & 0.038 \\
\hline \multicolumn{7}{|c|}{$6-311 \mathrm{G}^{* *}\left(-14.633376 E_{\mathrm{h}}\right)$} \\
\hline CMX-HW(1) & 0.274 & 0.274 & 1.528 & 0.004 & 0.002 & 1.265 \\
\hline CMX-HW(2) & 0.274 & 0.052 & 0.175 & 0.004 & 0.000 & 0.082 \\
\hline CMX-HW(3) & -0.049 & 0.005 & 0.069 & 0.004 & 0.000 & 0.036 \\
\hline CMX-HW(4) & -0.210 & 0.001 & 0.032 & 0.001 & 0.000 & 0.040 \\
\hline CMX-LT(4) & -0.207 & 0.002 & 0.031 & 0.002 & 0.001 & 0.040 \\
\hline
\end{tabular}

${ }^{a}$ using CC moments, Eq. (23), CMX-HW(1) is equal to CCSD or CCSDT.

${ }^{\mathrm{b}}$ using XCC moments, Eq. (16).

${ }^{c}$ CMX-HW(1) is equal to CISD or CISDT.

which has the same form as the original Horn-Weinstein function of Eq. (2), just with $\hat{H}$ replaced by $\bar{H}$. Accordingly, now one has connected moments of $\bar{H}$ defined by Eq. (23), and the CMX expansion may proceed along the same line as with the symmetric formulas. Although the similarity transformed Hamiltonian $\bar{H}$ is more than a mere two-particle operator, evaluation of the non-symmetric connected moments is, nevertheless, substantially simpler than (16). The reason 
is that, in opposite to (16), $\bar{H}^{k}$ always consists of finite number of terms, for finite $k$.

There are some important differences between (2) and (24), however. The most important one is that while $f(\eta)$ is monotonically decreasing, this statement does not hold for $\tilde{f}(\eta)$. It may happen therefore, that estimating $\tilde{f}(\infty)$ with low order derivatives does not improve the energy. Another interesting difference is that while the symmetric second moment, $f^{\prime}(0)$, being the distribution of the Hamiltonian, vanishes if and only if $\Phi$ is an exact eigenfunction, $\tilde{f}^{\prime}(0)$ may be zero due to several reasons. In particular, one may easily prove that it vanishes for a CCSD wave function provided that the amplitude equations have been exactly solved. Coming back to Eq. (20) we can write:

$$
\begin{aligned}
I_{2}^{C C} & =\langle H F|\bar{H} \bar{H}| H F\rangle-E_{C C}^{2} \\
& =\sum_{i}\left\langle H F|\bar{H}| H F_{i}\right\rangle\left\langle H F_{i}|\bar{H}| H F\right\rangle-E_{C C}^{2} \\
& =\sum_{i \neq 0}\left\langle H F|\bar{H}| H F_{i}\right\rangle\left\langle H F_{i}|\bar{H}| H F\right\rangle+E_{C C}^{2}-E_{C C}^{2}=0,
\end{aligned}
$$

because, terms with $i \leq 2$ in the last line vanish due to the fulfillment of CC equations Eq. (15), while for any $i>2$ the matrix elements $\left\langle H F|\bar{H}| H F_{i}\right\rangle$ vanish as well.

Consequently, no 'second order' correction to CCSD appears in this CC-CMX theory. This, on one hand, underlines the accuracy of the CCSD wave function, on the other hand it makes the evaluation of correction terms more difficult, as it necessitates going to higher $\operatorname{CMX}(\mathrm{n})$.

\section{Test Results}

For a CC reference we shall use "CC" for CMX-HW or CMX-LT expansions in which connected moments were calculated according to Eq. (23), whereas by "XCC" (expectation value CC) we denote results obtained from connected moments of Eq. (16). To test the aforementioned idea we applied a determinantal approach using the general CC-CI algorithm implemented recently [9]. With very little modifications, the latter code allows to calculate connected moments of all kinds we need.

In Tables 1 to 3 we display deviations of the CMX energies from FCI for model systems such as beryllium, $\mathrm{H}_{2} \mathrm{O}$, and $\mathrm{HF}$. Though the use of larger basis sets would be welcome, even results with the small bases used here allow to preliminary asses the performance of various CMX approaches, and eventually compare them to other recently suggested non-iterative corrections. We have calculated CMX expansions from CI wave functions as well.

Though Be atom is merely a four electron system, due to the $2 s^{2} / 2 p^{2}$ near-degeneracy, triple and quadruple substitutions are particularly important here. Extending the basis set, the role of higher excitations rises, of course. Such a trend is seen from Table 1. Unlike CISDT, the CCSDT 
Table 2: Deviations of the CMX energies (in $\mathrm{m} E_{\mathrm{h}}$ ) from the full CI energies for the $\mathrm{H}_{2} \mathrm{O}$ molecule $(\alpha=104.0 \mathrm{deg})$.

\begin{tabular}{|c|c|c|c|c|c|c|c|c|c|}
\hline \multirow{3}{*}{ Ref. } & \multicolumn{3}{|c|}{$\mathrm{R}=1.01 \AA$} & \multicolumn{3}{|c|}{$\mathrm{R}=1.5 \AA$} & \multicolumn{3}{|c|}{$\mathrm{R}=2.0 \AA$} \\
\hline & \multicolumn{2}{|c|}{ CCSD } & \multirow[t]{2}{*}{ CISD } & \multicolumn{2}{|c|}{ CCSD } & \multirow[t]{2}{*}{ CISD } & \multicolumn{2}{|c|}{ CCSD } & \multirow[t]{2}{*}{ CISD } \\
\hline & $\mathrm{CC}$ & $\mathrm{XCC}$ & & $\mathrm{CC}$ & $\mathrm{XCC}$ & & $\mathrm{CC}$ & $\mathrm{XCC}$ & \\
\hline STO-3G/FCI ${ }^{\mathrm{a}}$ & \multicolumn{3}{|c|}{$-83.736245 E_{\mathrm{h}}$} & \multicolumn{3}{|c|}{$-82.218671 E_{\mathrm{h}}$} & \multicolumn{3}{|c|}{$-81.401484 E_{\mathrm{h}}$} \\
\hline CMX-HW(1) & 0.145 & 0.117 & 0.990 & 0.378 & 0.175 & 4.045 & 0.417 & 0.119 & 9.354 \\
\hline CMX-HW(2) & 0.145 & 0.029 & 0.167 & 0.378 & 0.066 & 0.831 & 0.417 & 0.073 & 2.411 \\
\hline CMX-HW(3) & 0.146 & 0.029 & 0.071 & 0.383 & 0.040 & 0.319 & 0.423 & 0.060 & 0.922 \\
\hline CMX-HW(4) & 0.158 & 0.030 & 0.066 & 0.409 & 0.034 & 0.321 & 0.452 & 0.063 & 0.935 \\
\hline CMX-LT(4) & 0.189 & 0.009 & 0.050 & 0.505 & 0.017 & 0.327 & 0.565 & 0.071 & 0.963 \\
\hline $3-21 \mathrm{G} / \mathrm{FCI}^{\mathrm{a}}$ & \multicolumn{3}{|c|}{$-84.434106 E_{\mathrm{h}}$} & \multicolumn{3}{|c|}{$-82.923660 E_{\mathrm{h}}$} & \multicolumn{3}{|c|}{$-82.112076 E_{\mathrm{h}}$} \\
\hline CMX-HW(1) & 1.886 & 1.670 & 6.372 & 3.953 & 3.511 & 12.422 & 7.552 & 6.584 & 25.057 \\
\hline CMX-HW(2) & 1.886 & 0.483 & 1.705 & 3.953 & 1.062 & 4.527 & 7.552 & 2.145 & 10.997 \\
\hline CMX-HW(3) & 2.071 & 0.195 & 0.813 & 4.250 & 0.495 & 2.519 & 7.996 & 1.137 & 6.209 \\
\hline CMX-HW(4) & 1.448 & 0.168 & 0.601 & 2.777 & 0.463 & 2.009 & 5.620 & 0.975 & 5.130 \\
\hline CMX-LT(4) & 1.034 & 0.142 & 0.436 & 1.694 & 0.431 & 1.591 & 3.853 & 0.791 & 4.259 \\
\hline
\end{tabular}

${ }^{\text {a} E l e c t r o n i c ~ e n e r g y ~}$

covers not only the major portion of triples, but via disconnected $T_{2} T_{2}$ clusters also the quadruple excitations. Therefore, results with CCSDT reference are very close to the full CI limit.

As one can see, the superiority of XCC-CMX is obvious, both with respect to CC-CMX and CMX with a CISD (or CISDT) reference. This has been, in general, expected. The question to answer was just how much would these approaches differ. The reason is simple. Formally, the reference function for XCC-CMX includes all possible excited determinants that can be created with respect to one-electron approximation ground state. Hence, in a sense, one calculates the full CI like energy already in $I_{1}$. Let us note that $I_{1}$ is an upper bound and size extensive at the same time. With limited CI, one starts with a wave function that is not size extensive (linked). With such a reference function the moments $I_{k}$ are actually not strictly connected, and the approximate energies are not size extensive. Nevertheless, we have included the CI wave function based CMX energies for curiosity. Despite the aforementioned drawbacks of limited CI, the convergence towards the FCI limit for beryllium has been better with CISD reference than using the CC moments (23) from the CCSD reference. 
Table 3: Deviations of the CMX energies (in $\mathrm{m} E_{\mathrm{h}}$ ) from the full CI energies for the HF molecule.

\begin{tabular}{|c|c|c|c|c|c|c|c|c|c|}
\hline \multirow{3}{*}{ Ref. } & \multicolumn{3}{|c|}{$\mathrm{R}=1.0 \AA$} & \multicolumn{3}{|c|}{$\mathrm{R}=1.5 \AA$} & \multicolumn{3}{|c|}{$\mathrm{R}=2.0 \AA$} \\
\hline & \multicolumn{2}{|c|}{ CCSD } & \multirow[t]{2}{*}{ CISD } & \multicolumn{2}{|c|}{ CCSD } & \multirow[t]{2}{*}{ CISD } & \multicolumn{2}{|c|}{ CCSD } & \multirow[t]{2}{*}{ CISD } \\
\hline & $\mathrm{CC}$ & $\mathrm{XCC}$ & & $\mathrm{CC}$ & $\mathrm{XCC}$ & & $\mathrm{CC}$ & $\mathrm{XCC}$ & \\
\hline $6-31 \mathrm{G} / \mathrm{FCI}^{\mathrm{a}}$ & \multicolumn{3}{|c|}{$-104.877215 E_{\mathrm{h}}$} & \multicolumn{3}{|c|}{$-103.201083 E_{\mathrm{h}}$} & \multicolumn{3}{|c|}{$-102.348497 E_{\mathrm{h}}$} \\
\hline CMX-HW(1) & 1.233 & 0.958 & 5.865 & 3.379 & 2.861 & 11.730 & 7.545 & 5.809 & 24.322 \\
\hline CMX-HW(2) & 1.233 & 0.386 & 1.234 & 3.379 & 1.120 & 3.610 & 7.545 & 2.572 & 9.615 \\
\hline CMX-HW(3) & 1.296 & 0.110 & 0.520 & 3.480 & 0.477 & 1.859 & 7.695 & 1.745 & 5.117 \\
\hline CMX-HW(4) & 0.933 & 0.092 & 0.383 & 2.287 & 0.419 & 1.545 & 5.620 & 0.497 & 4.508 \\
\hline CMX-LT(4) & 0.610 & 0.077 & 0.231 & 0.925 & 0.352 & 1.179 & 3.002 & -1.941 & 3.827 \\
\hline
\end{tabular}

${ }^{a}$ Electronic energy

Unlike CISD, CCSD reference and the CC moments of the Hamiltonian are precisely size extensive, but the first moments are not strictly upper bound. Consequently, as mentioned in the previous section, the pattern of $\tilde{f}(\eta)(24)$ may not be monotonic as one would like to have. This most probably explains the "worst" performance of the CC moments, from the three investigated approaches for beryllium. As soon as we start from CCSDT wave function - which is almost "exact" - CMX through CC moments outperforms the CISDT based CMX.

In Tables 2 and 3 we have included $\mathrm{H}_{2} \mathrm{O}$ and $\mathrm{HF}$ with gradually extended bond lengths, $\mathrm{i}$. e. with higher and higher portion of non-dynamical correlation effects. Also here, one sees the "best" performance of XCC based CMX. Up to CMX(4), the CMX-HW does not provide results too different from CMX-LT. Consequently one could hardly prefer any of those two. Anyway, a computationally practical level of approximation would be more or less $\operatorname{CMX}(3)$ at which both HW and LT expansions are identical.

The relatively large difference between CMX-HW(4) and CMX-LT(4) for HF at $2.0 \AA$ may be due to the closeness to a singularity. This problem has been analysed by Mancini et al. [46], who showed that in HW expansion the singularities are canceled out in various orders of CMX. Our initial experience revealed that the connected moments and any intermediate quantities can differ in many orders of magnitude, and the numerical precision can be easily affected (not only) due to the closeness of a singularity.

As to the concluding assessment, let us recall that our test calculations showed that of the two coupled cluster based CMX approaches only XCC based CMX could be competitive with the recently proposed non-iterative corrections such as renormalized $\operatorname{CCSD}(\mathrm{T})$ [31] or perturbative corrections to coupled cluster energies [12,34]. The disadvantage of the XCC CMX moments is that 
easily factorizable expansions for the expectation values given by Eq. (16) are non-terminating, and calculation through a determinantal approach based on the CI type matrix elements is computationally extremely demanding. More numerical experience is needed in order to recommend further development along the line to truncate the infinite expansions of (16).

\section{Acknowledgments}

This work has been supported by the Slovak grant agency VEGA (project 2/7203/20), and Hungarian grants FKFP 0165/1999-0144/2000 and OTKA 030435-35094. This work has also benefitted from the COST Chemistry action D9/WG013.

\section{References}

1. Coester, F. Nucl. Phys. 1958, 7, 421; Coester, F. and Kümmel, H. Nucl. Phys. 1960. 17, 477.

2. Č́žzek, J. J. Chem. Phys. 1966, 45, 4256.

3. Č́žek, J. Adv. Chem. Phys. 1969 14, 35.

4. Purvis, G. D. and Bartlett, R. J. J. Chem. Phys. 1982, 76, 1910.

5. Noga, J. and Bartlett, R. J. J. Chem. Phys. 1987 86, 7071; 1988, 89, 3401 (E).

6. Scuseria, G. E. and Schaefer III, H. F. Chem. Phys. Lett. 1988, 152, 382.

7. Oliphant, N. and Adamowicz, L. J. Chem. Phys. 1991, 95, 6645.

8. Kucharski, S. A. and Bartlett, R. J. Theor. Chim. Acta 1991, 80, 387; J. Chem. Phys. 1992, 97, 4282 .

9. Kállay, M. and Surján, P. J. Chem. Phys. 2000, 113, 1359.

10. Kállay, M. and Surján, P. J. Chem. Phys. 2001, 115, 2945.

11. Olsen, J. J. Chem. Phys. 2000, 113, 7140.

12. Hirata, S.; Nooijen, M.; Grabowski, I. and Bartlett, R. J. J. Chem. Phys. 2001, 114, 3919; 2001, 115, $3967(\mathrm{E})$.

13. Lee, Y. S.; Kucharski, S. A. and Bartlett, R. J. J. Chem. Phys. 1984, 81, 5906.

14. Urban, M.; Noga, J.; Cole, S. J. and Bartlett, R. J. J. Chem. Phys. 1985, 83, 404.

15. Noga, J.; Bartlett, R. J. and Urban, M. Chem. Phys. Lett. 1987, 134, 126.

16. Kucharski, S. A. and Bartlett, R. J. Chem. Phys. Lett. 1989, 158, 550.

17. Raghavachari, K.; Trucks, G. W.; Pople, J. A. and Head-Gordon, M. Chem. Phys. Lett. 1989, 15\%, 479.

18. Noga, J.; Kutzelnigg, W. and Klopper, W. Chem. Phys. Lett. 1992, 199, 497.

19. Raghavachari, K. J. Chem. Phys. 1985, 82, 5761. 
20. Raghavachari, K.; Pople, J. A.; Replogle, E. S. and Head-Gordon, M. J. Phys. Chem. 1990, 94, 5579 .

21. Bartlett, R. J.; Watts, J. D.; Kucharski, S. A. and Noga, J. Chem. Phys. Lett. 1990, 165, 513.

22. Piecuch, P. and Paldus, J. Theor. Chim. Acta 1990, 78, 65; Piecuch, P.; Tobola, R. and Paldus, J. Int. J. Quantum Chem. 1995, 55, 133.

23. Kucharski, S. A. and Bartlett, R. J. Chem. Phys. Lett. 1993, 206, 574.

24. Deegan, M. J. O. and Knowles, P. J. Chem. Phys. Lett. 1994, 227, 321.

25. Stanton, J. and Gauss, J. J. Chem. Phys. 1995, 103, 1064; Theor. Chim. Acta 1996, 93, 303; 1997, 95, 97 (E); Crawford, T. D. and Stanton, J. F. Int. J. Quantum Chem. 1998, 70, 601 .

26. Christiansen, O.; Koch, H. and Jørgensen, P. J. Chem. Phys. 1996, 105, 1451.

27. Crawford, T. D.; Lee, T. J. and Schaefer III, H. F. J. Chem. Phys. 1997, 10\%, 7943.

28. Kucharski, S. A. and Bartlett, R. J. J. Chem. Phys. 1998, 108, 5243.

29. Kucharski, S. A. and Bartlett, R. J. J. Chem. Phys. 1998, 108, 5255.

30. Kowalski K. and Piecuch, P. in Computational Chemistry: Reviews of Current Trends; Leszcynski, J., Ed.; World Scientific: Singapore, 2000; Vol 5., p. 1.

31. Kowalski, K. and Piecuch, P. J. Chem. Phys. 2000, 113, 18.

32. Kowalski, K. and Piecuch, P. J. Chem. Phys. 2001, 115, 2966.

33. Kowalski, K. and Piecuch, P. J. Molec. Struct. (THEOCHEM) 2001, 547, 191.

34. Gwaltney, S. R. and Head-Gordon, M. Chem. Phys. Lett. 2000, 323, 21; Gwaltney, S. R.; Sherrill, C. D.; Head-Gordon, M. and Krylov, A. I. J. Chem. Phys. 2000, 113, 3548; Gwaltney, S. R. and Head-Gordon, M. J. Chem. Phys. 2001, 115, 2014.

35. Meissner, L. and Bartlett, R. J. J. Chem. Phys. 2001, 115, 50.

36. Kucharski, S. A.; Noga, J. and Bartlett, R. J. J. Chem. Phys. 1989, 90, 7282.

37. Noga, J. and Pluta, T. Chem. Phys. Lett. 1997, 264, 101.

38. Olsen, J.; Christiansen, O.; Koch, H. and Jorgensen, P. J. Chem. Phys. 1996, 105, 5082.

39. Jankowski, K. and Kowalski, K. J. Chem. Phys. 1999, 111, 2952.

40. Kowalski, K. and Piecuch, P. J. Chem. Phys. 2000, 113, 5644; Piecuch, P.; Kucharski, S. A.; Špirko, V. and Kowalski, K. J. Chem. Phys. 2001, 115, 5796; Kowalski, K. and Piecuch, P. Chem. Phys. Lett. 2001, 344, 165; Piecuch, P.; Kucharski, S. A. and Kowalski, K. Chem. Phys. Lett. 2001, 344, 176.

41. Stanton, J. F. Chem. Phys. Lett. 1997, 281, 130.

42. Cioslowski, J. Phys. Rev. Lett. 1987, 58, 83.

43. Cioslowski, J.; Kertész, M.; Surján, P. and Poirier, R. A. Chem. Phys. Lett. 1987, 138, 516. 
44. Cioslowski, J. Int. J. Quantum Chem. 1987, S21, 563.

45. Knowles, P. Chem. Phys. Lett. 1987, 134, 512.

46. Mancini, J. D.; Zhou, Y. and Meier, P. F. Int. J. Quantum Chem. 1994, 50, 101.

47. Yoshida, T. and Iguchi, K. Chem. Phys. Lett. 1988, 143, 329; J. Chem. Phys. 1989, 91, 4249 .

48. Wolinski, K. and Pulay, P. J. Chem. Phys. 1989, 90, 3647.

49. Surján, P. and Szabados, Á. to be published

50. Horn, D. and Weinstein, M. Phys. Rev. D 1984, 30, 1256.

51. Lanczos, C. J. J. Res. Natl. Bur. Std. 1950 45, 55

52. Bartlett, R. J. and Noga, J. Chem. Phys. Lett. 1988, 150, 29. 